\title{
Kaon Physics at CERN: recent results from the NA48/2 experiment
}

\author{
Cristina Biino*广 \\ INFN Torino \\ E-mail: cristina.biinoeto.infn.it
}

\begin{abstract}
Semileptonic kaon decays offer the most precise determination of the CKM matrix element IVusI. The experimental precision is however limited by the knowledge of the form factors of these decays. The NA48/2 experiment presents high precision preliminary measurements of the form factors of the semileptonic decays of charged kaons $\left(K^{ \pm} \rightarrow \pi^{0} l^{ \pm} v\right)$, based on 4.3 million Ke3 and 2.5 million $K \mu 3$ decays, both with negligible background.

At the same time a large sample of more than a million charged kaon decays to Ke4 final state (for both charged, $K^{ \pm} \rightarrow \pi^{+} \pi^{-} e^{ \pm} v$, and neutral, $K^{ \pm} \rightarrow \pi^{0} \pi^{0} e^{ \pm} v$, mode) has been collected, allowing a detailed study of $\pi \pi$ scattering and hadronic form factors. Here we present a much improved determination of the Branching Fraction for both charged and neutral modes and Form Factor for the charged mode.
\end{abstract}

36th International Conference on High Energy Physics,

July 4-11, 2012

Melbourne, Australia

\footnotetext{
* Speaker.

${ }^{\dagger}$ Representing the NA48/2 collaboration.
} 


\section{The NA48 experiment at CERN}

The original goal of the NA48 experiment at CERN was to measure direct CP Violation (CPV) in the neutral kaon system [1] but more recently NA48/2 has started working with charged kaon beams to measure CPV asymmetry in the $K^{ \pm}$decays to three pions [2]. The NA48/2 experiment has been collecting data from charged kaon decays in the years 2003 and 2004. Positive and negative kaons were simultaneously produced from the $400 \mathrm{GeV} / \mathrm{c}$ proton beam, extracted from CERN SPS. Charged kaons with a momentum range of $p_{K}=(60 \pm 3) \mathrm{GeV} / \mathrm{c}$ were selected by two systems of dipole magnets, focusing quadrupoles, muon sweepers and collimators The layout of beams and detectors is shown in Fig.1.

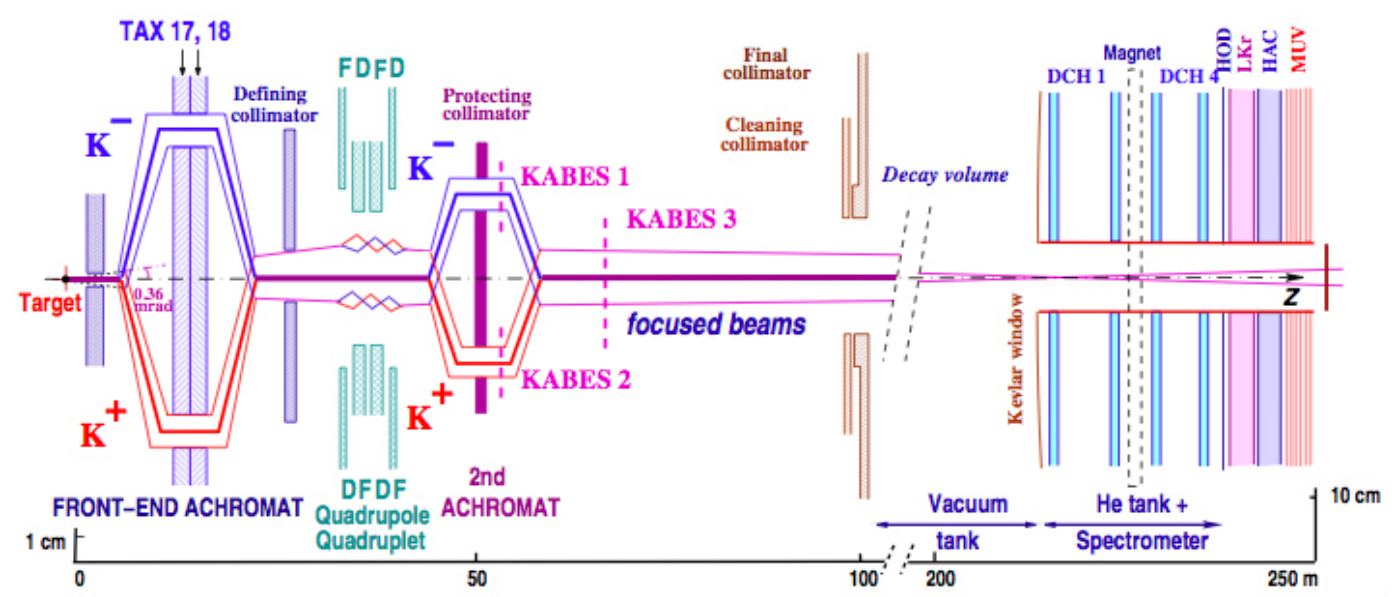

Figure 1: Schematic side view of the NA48/2 beamline and detectors.

A detailed detector description can be found in [3]. The main components of the NA48/2 detector are a magnetic spectrometer, composed by four drift chambers, a dipole magnet deflecting the charged particles in the horizontal plane and providing a momentum resolution of $1.4 \%$ for 20 $\mathrm{GeV} / \mathrm{c}$ charged tracks, and a hodoscope to provide trigger and time resolution for charged particles; a liquid krypton electromagnetic calorimeter $(\mathrm{LKr})$ with an energy resolution of about $1 \%$ for $20 \mathrm{GeV}$ photons and electrons and very good time resolution. The muon veto system (MUV) is essential to distinguish muons from pions. It consists of planes of alternating horizontal and vertical scintillator strips, shielded by an iron wall.

\section{Semileptonic Kaon Decays: $\mathrm{K} \mu 3$ and Ke3}

Semileptonic kaon decays $K^{ \pm} \rightarrow \pi^{0} l^{ \pm} v(\mathrm{Kl} 3,1=\mu$,e) provide the most accurate and theoretically cleanest way to measure the CKM matrix element $\left|V_{u s}\right|$ [4]. In addition, tight constraints on new physics can be given by testing the V-A structure in K $\mu 3$ decays [5] or lepton universality. The experimental precision is however limited by the knowledge of the Form Factors (FF) of these decays, since the FF enter both the phase space integral and the detector acceptances. The hadronic matrix element of $\mathrm{Kl} 3$ decays is described by two dimensionless FF $f_{ \pm}(\mathrm{t})$, which depend on the squared four-momentum $t=\left(p_{K}-p_{\pi}\right)^{2}$ transferred to the lepton system. Form factors are usually formulated to express the vector $\left(1^{-}\right)$and scalar $\left(0^{+}\right)$exchange contributions, using the $f_{+}$and $f_{0}$ functions, defined as: $f_{0}(t)=f_{+}(t)+t /\left(m_{K}^{2}-m_{\pi}^{2}\right) \times f_{-}(t)$. The contribution of $f_{-}$can be neglected 
in Ke3 decays being proportional to the lepton mass squared. By construction $f_{0}(0)=f_{+}(0)$ and since $f_{+}(0)$ is not directly measurable (compute by theory: i.e. lattice QCD or ChPT), it is customary to factor out and to normalize all FF to this quantity, so that: $\bar{f}_{+}(t)=f_{+}(t) / f_{+}(0)$ and $\bar{f}_{0}(t)=f_{0}(t) / f_{+}(0)$.

To describe the form factors, we have been using two different parametrizations. The well known and most used Taylor expansion, called also quadratic parametrization, it is an expansion in the momentum transfer: $\bar{f}_{+, 0}(t)=\left(1+\lambda_{+, 0}^{\prime}\left(t / m_{\pi}^{2}\right)+1 / 2 \lambda_{+, 0}^{\prime \prime}\left(t / m_{\pi}^{2}\right)^{2}\right)$ where $\lambda^{\prime}$ and $\lambda^{\prime \prime}$ are the slope and the curvature of the FF, respectively. The disadvantage of this parametrization is related to the strong correlations between the parameters. To reduce the number of parameters and to add a physical motivation we use the pole parametrization: $\bar{f}_{+, 0}(t)=m_{V, S}^{2} /\left(m_{V, S}^{2}-t\right)$. This parametrization assumes the dominance exchange of a single vector or scalar resonance $K^{\star}$ with spin parity $1^{-}$or $0^{+}$. The resonance pole masses $m_{V}, m_{S}$ are the only free parameters.

The data used for the $\mathrm{Kl} 3$ form factors analysis were collected in a dedicated run with a special minimum bias trigger which required one or more tracks in the magnetic spectrometer and an energy deposit of at least $10 \mathrm{GeV} / \mathrm{c}$ in the electromagnetic calorimeter. The data selection requires one track in the magnetic spectrometer and a time coincidence with at least two clusters in the electromagnetic calorimeter from the $\pi^{0}$ decay. To identify a track as the electron we require 0.95 $<\mathrm{E} / \mathrm{p}<1.05$, where $\mathrm{E}$ is the energy deposited in the e.m. calorimeter and $\mathrm{p}$ is the momentum measured in the magnetic spectrometer, and no signal in the MUV system. To identify the track as a muon we require an associated hit in the MUV system and $E / p<0.2$. Finally the missing mass squared is required to satisfy $m_{\text {miss }}^{2}<\left(10 \mathrm{MeV} / c^{2}\right)^{2}$ under a $K^{ \pm}$decay hypothesis.

For $K^{ \pm} e 3$, the only background contributing is from $K^{ \pm} \rightarrow \pi^{ \pm} \pi^{0}$. An additional cut in the transverse momentum of the event reduces the background to less than $0.1 \%$ while loosing only $3 \%$ of the signal. For $K^{ \pm} \mu 3$, the background from $K^{ \pm} \rightarrow \pi^{ \pm} \pi^{0}$ with the charged pion decaying in flight was suppressed by using a combined requirement on the invariant mass $m_{\pi^{ \pm} \pi^{0}}$ (under $\pi^{ \pm}$ hypothesis) and on the $\pi^{0}$ transverse momentum. This cut reduces the background contamination to $0.5 \%$ but causes a loss of about $24 \%$ of the signal statistics.

The NA48/2 experiment selected sample of candidate decays amounts to 4.0 million Ke3 and 2.5 million $K \mu 3$. We apply corrections for acceptance and radiative effects. To extract the FF a two-dimensional fit is performed in $5 \times 5 \mathrm{MeV}^{2}$ cells in the Dalitz plot of Pion energy vs Lepton energy, computed in the kaon rest frame. The kaon energy calculation is done by assuming no transverse component of the kaon momentum, which leaves only two possible solutions for the longitudinal component of the neutrino momentum. The solution that fits better the known kaon beam momentum of $60 \mathrm{GeV} / \mathrm{c}$ is used. Cells outside or crossing the border of the physical region of the Dalitz plot are not used in the fit. The preliminary fit results for the K13 form factors in the quadratic and the pole parametrizations are given in Table 1.

The systematic errors are evaluated by changing the cuts by small amounts. We applied also variations to the resolution of pion and muon energies, we varied $\pi \rightarrow \mu$ background and took into account the differences in the results of two independent analysis. In Fig. 2 are shown our combined $\mathrm{Kl} 3$ quadratic fit results together with recent results by other experiments (KLOE, KTeV and NA48 for neutral kaons and ISTRA+ for $K^{-}$). The $68 \%$ confidence levels contours are shown.

NA48/2 is the first experiment measuring the FF using both $K^{+}$and $K^{-}$. In $K \mu 3$ the result is 


\begin{tabular}{|c||c|c|c||c|c|}
\hline & $\lambda_{+}^{\prime}\left[10^{-3}\right]$ & $\lambda_{+}^{\prime \prime}\left[10^{-3}\right]$ & $\lambda_{0}\left[10^{-3}\right]$ & $M_{V}\left[\mathrm{MeV} / \mathrm{c}^{2}\right]$ & $M_{S}\left[\mathrm{MeV} / \mathrm{c}^{2}\right]$ \\
\hline \hline$K^{ \pm} e 3$ & $27.2 \pm 0.7 \pm 1.1$ & $0.7 \pm 0.3 \pm 0.4$ & & $879 \pm 3 \pm 7$ & \\
\hline$K^{ \pm} \mu 3$ & $26.3 \pm 3.0 \pm 2.2$ & $1.2 \pm 1.1 \pm 1.1$ & $15.7 \pm 1.4 \pm 1.0$ & $873 \pm 8 \pm 9$ & $1183 \pm 31 \pm 16$ \\
\hline \hline combined & $26.98 \pm 1.11$ & $0.81 \pm 0.46$ & $16.23 \pm 0.95$ & $877 \pm 6$ & $1176 \pm 31$ \\
\hline \hline
\end{tabular}

Table 1: Preliminary form factors results for the quadratic and the pole parametrizations. The first error is statistical, the second systematic. For the combined results the errors are combined.
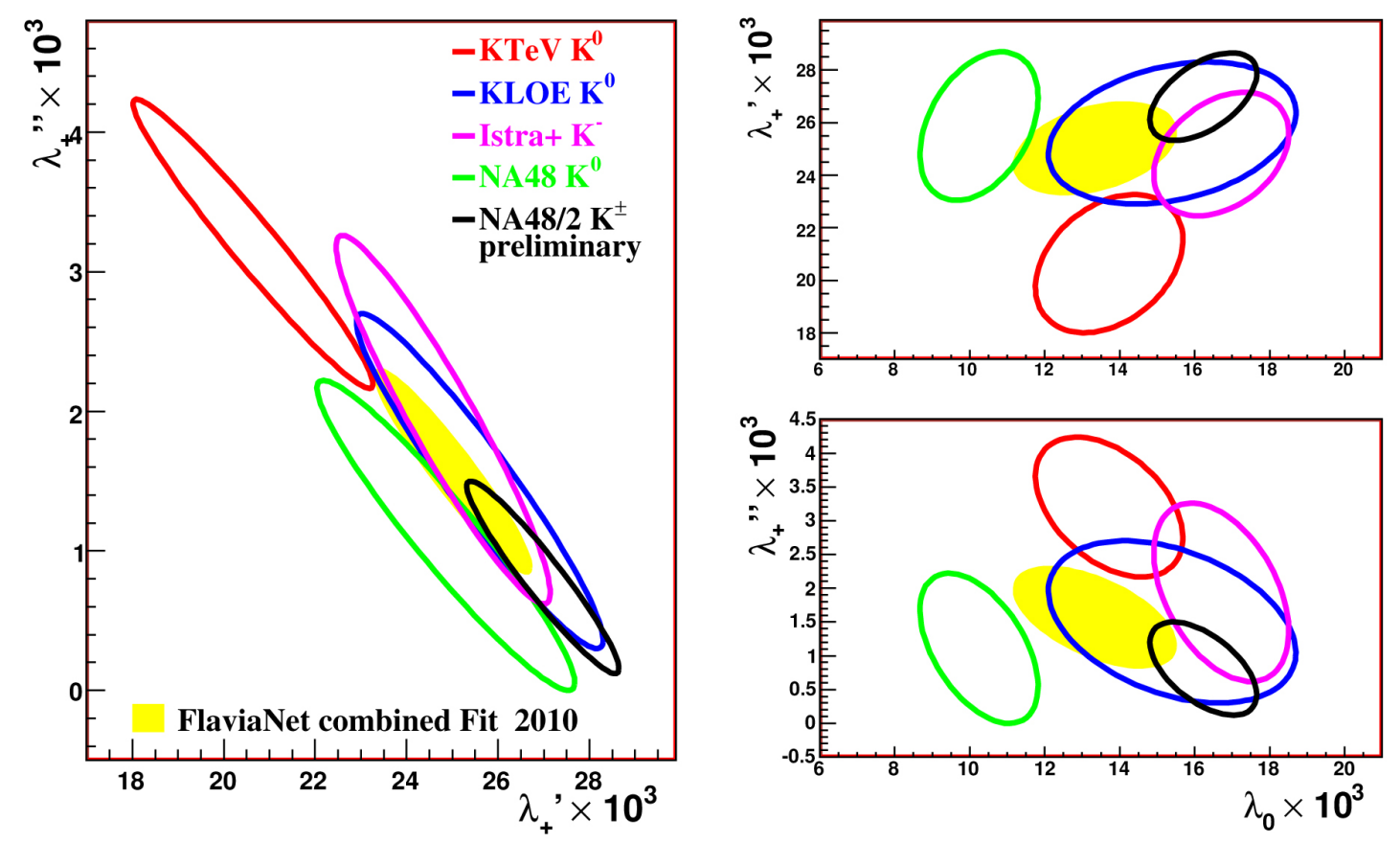

Figure 2: Combined quadratic fit results for $K^{ \pm} 13$ decays, our preliminary result is shown in black. For comparison the combined fit from the Flavia net kaon working group is also shown [4], in yellow. The ellipses are the $68 \%$ confidence levels contours.

dominated by the statistical error, for Ke3 by the systematic. Our preliminary results are in agreement with previous results (except $K^{0} \mu 3$ from NA48 [6]), they have the smallest error for the Ke3 and are very competitive with other $K \mu 3$ results. The NA48/2 $K^{ \pm} e 3$ and $K^{ \pm} \mu 3$ are in agreement within each other and our combined results are competitive with the current world average.

\section{Measurement of Ke4 decay}

The main aim of NA48/2experiment was the search for direct CP violation in decays of charged kaons into three pions. The trigger therefore was designed to efficiently select events with three charged tracks as well as $K^{ \pm} \rightarrow \pi^{ \pm} \pi^{0} \pi^{0}$ events.

Thanks to the very big sample of data collected for both $K^{+}$and $K^{-}$many other interesting measurements have been performed with unprecedented precision. In particular, the measurements of $\pi \pi$ scattering lengths have ben obtained using two different and independent approaches: a measurement of $\pi \pi$ scattering lengths from cusp effect in the decay $K^{ \pm} \rightarrow \pi^{ \pm} \pi^{0} \pi^{0}$ [7] and a 
complementary measurement, with different systematics but with similar precision, using the rare Ke4 decays $K^{ \pm} \rightarrow \pi^{+} \pi^{-} e^{ \pm} v$ [8] [9]. The combination of both measurements provides one of the most stringent test of Chiral Perturbation Theory (ChPT).

The Ke4 decay is a rare decay with a branching fraction of about $4 \times 10^{-5}$. A sample of more than one million charged $K^{ \pm} \rightarrow \pi^{+} \pi^{-} e^{ \pm} \nu$ and 60,000 neutral $K^{ \pm} \rightarrow \pi^{ \pm} \pi^{0} \pi^{0}$ Ke4 decays candidates with less than one percent background contamination has been collected by the NA48/2 experiment, allowing a detailed study of the decay properties. In this paper we report on the recent form factors and branching ratio measurements.

The Ke4 rate is measured relative to the abundant $K 3 \pi$ normalization channel, $K^{ \pm} \rightarrow \pi^{ \pm} \pi^{+} \pi^{-}$ for the charged mode and $K^{ \pm} \rightarrow \pi^{ \pm} \pi^{0} \pi^{0}$ for the neutral mode. As the topologies of the two modes are similar to their normalization mode the two samples (signal and normalization) are collected concurrently using the same trigger logic and a common event selection was used as much as possible. This leads to partial cancellation of the systematic effects induced by imperfect kaon beam description, local detector inefficiencies and trigger inefficiency. The Ke4 rate relative to $K 3 \pi$ normalization and the Ke4 branching ratio (BR) are obtained as: $\Gamma(K e 4) / \Gamma(K 3 \pi)=$ $\left(N_{S}-N_{B}\right) / N_{N} \times A_{N} / A_{S} \times \varepsilon_{N} / \varepsilon_{S}$ and $B R(K e 4)=\left(N_{S}-N_{B}\right) / N_{N} \times A_{N} / A_{S} \times \varepsilon_{N} / \varepsilon_{S} \times B R(K 3 \pi)$ where $N_{S}, N_{B}, N_{N}$ are the numbers of signal, background and normalization candidates, $A_{S, N}$ and $\varepsilon_{S, N}$ are the acceptance and trigger efficiencies for the signal and the normalization channels. $K^{-} e 4$ rate has never been measured and, since no difference is expected from the $K^{+} e 4$ rate, a comparison of the separate measurements was used as a consistency check. The analysis includes radiative events and is a loosen version of the one used to study $\pi \pi$ scattering [9]. The values used for the normalization channels are the world average [10]: $B R\left(K^{ \pm} \rightarrow \pi^{ \pm} \pi^{+} \pi^{-}\right)=(5.59 \pm 0.04) \%$, for the charged mode, and $B R\left(K^{ \pm} \rightarrow \pi^{ \pm} \pi^{0} \pi^{0}\right)=(1.761 \pm 0.022) \%$, for the neutral mode.

For the charged mode we obtain: $N_{S}=1.11 \times 10^{6}$ events and $N_{N}=1.9 \times 10^{9}$ events with a background at $0.95 \%$ level of the Ke $4 ; A_{N}, A_{S}, \varepsilon_{N}, \varepsilon_{S}$ are $18.16 \%, 23.97 \%, 98.5 \%$ and $97.7 \%$ each. The final results are a weighted average of 16 values obtained in eight independent data subsamples and for both kaon charges : $B R\left(K^{+} e 4\right)=(4.255 \pm 0.008) \times 10^{-5} ; B R\left(K^{-} e 4\right)=(4.261 \pm 0.011) \times$ $10^{-5} ; B R\left(K^{ \pm} e 4(+-)\right)=\left(4.257 \pm 0.004_{\text {stat }} \pm 0.016_{\text {syst }} \pm 0.031_{\text {ext }}\right) \times 10^{-5}$. The fit $\chi^{2}$ is 15.85 for 15 degrees of freedom, when including the time-dependent errors only. The relative $0.8 \%$ precision of the measurement is dominated by the external uncertainty from the normalization mode.

In the past, without the knowledge of the branching ratio value, we could present only form factors relative to a common normalization [9]. Now the overall form factor normalization $f_{s}=$ $\left(5.705 \pm 0.003_{\text {stat }} \pm 0.017_{\text {exp }} \pm 0.031_{\text {ext }}\right)$ is obtained with a total relative precision of $0.6 \%$.

For the neutral mode we obtain: $N_{S}=44,909$ events and $N_{N}=1.71 \times 10^{6}$ events with a background at $1.3 \%$ level of Ke $4 ; A_{N}$ and $A_{S}$ are $1.77 \%$ and $4.11 \%$; both trigger efficiencies are in the range $92-98 \%$. The preliminary result is a weighted average of 7 values obtained in independent data subsamples: $B R\left(K^{ \pm} e 4(00)\right)=\left(2.595 \pm 0.012_{\text {stat }} \pm 0.024_{\text {syst }} \pm 0.032_{\text {ext }}\right) \times 10^{-5}$. The fit $\chi^{2}$ is 5.32 for 6 degrees of freedom. First approach to the $\operatorname{Ke} 4(00) F_{s}$ form factor measurements shows consistency with the $F_{S} \mathrm{Ke} 4(+-)$ form factor.

\section{Conclusion}

Semileptonic kaon decays offer the most precise determination of the CKM matrix element 
IVusl. The experimental precision is however limited by the knowledge of the form factors of this decay, since these enter both the phase space integral and the detector acceptances. The NA48/2 experiment presents new preliminary high precision measurements of the form factors of the semileptonic decays of charged kaons $\left(K^{ \pm} \rightarrow \pi^{0} l^{ \pm} v\right)$, based on 4.3 million $\mathrm{Ke} 3$ and 2.5 million $K \mu 3$ decays, both with negligible background. The result matches the precision of the current world average on the vector and scalar form factors and allows to significantly reduce the form factor uncertainty on IVusl. In addition, the comparison of both channels sets tight constraints on lepton flavour violation and other possible new physics.

The NA48/2 collaboration has also accumulated more than one million K charged decays Ke4(+-) ( $\left.K^{ \pm} \rightarrow \pi^{+} \pi^{-} e v\right)$ leading to an improved determination of the Branching Fraction by a factor of 3 with respect to the world average and detailed Form Factor studies, evaluated in absolute value and energy dependence. Concurrently, 60,000 semi-leptonic K charged decays Ke4(00) $\left(K^{ \pm} \rightarrow \pi^{0} \pi^{0} e v\right.$ ) have been analyzed, increasing the world available statistics by several orders of magnitude. Background contamination below the percent level and very good $\pi^{0}$ reconstruction allow the first accurate measurement of the Branching Fraction and decay Form Factor at the percent level. The achieved precision makes possible the observation of small effects such as $\pi^{+} \pi^{-}$ to $\pi^{0} \pi^{0}$ re-scattering below the $2 \mathrm{~m}\left(\pi^{+}\right)$threshold. Both Ke4 modes decay properties bring new precise inputs to Low Energy QCD studies and can provide strong tests of ChPT predictions.

While the above analyses will appear shortly with final results, future prospects include the observation of several thousand decays in similar muonic modes $\mathrm{K} \mu 4(00)$ (never observed) and K $\mu$ 4(+-) (7 events observed). Such poorly known modes could be studied also in the forthcoming NA62 experiment currently under construction. The NA62 experiment, using the same beam line and detector of NA48/2, has collected data in 2007 for the measurement of $R_{K}=\Gamma(K e 2) / \Gamma(K \mu 2)$ and is preparing for the future $K^{+} \rightarrow \pi^{+} v \bar{v}$ rare decay experiment. The already collected data contains $K^{+} e 3$ and $K^{+} \mu 3$ samples of 40 and 20 millions events, respectively. There has been also a special $K_{L}$ data taking providing $K^{0} e 3$ and $K^{0} \mu 3$ samples of about 4 millions events. With these statistics NA62 will obtain high precision measurements of the form factors of all Kl3 channels, providing important inputs to further reduce the uncertainty on IVusl.

\section{References}

[1] V. Fanti et al. [NA48 Collab.], Phys. Lett. B 465 (1999) 335.

[2] J.R. Batley et al. [NA48/2 Collab.], Phys. Lett. B 649 (2007) 349.

[3] V. Fanti et al. [NA48 Collab.], Nucl. Instr. Meth. A 574 (2007) 433.

[4] M. Antonelli et al. (Flavianet Kaon WG), E. Physi. J. C 69 (2010) 399.

[5] V. Bernard, M. Oertel, E. Passemar and J. Stern, Phys. Rev. Lett. B 638 (2006) 480.

[6] A. Lai et al. [NA48 Collab.], Phys. Lett. B 647 (2007) 341.

[7] J.R. Batley et al. [NA48/2 Collab.], Phys. Lett. B bf 633 (2006) 173.

[8] J.R. Batley et al. [NA48/2 Collab.], Eur./ Phys. J. C 54 (2008) 411.

[9] J.R. Batley et al. [NA48/2 Collab.], Eur./ Phys. J. C 70 (2010) 635.

[10] K. Nakamura et al. (PDG), J. Phys. G37 (2010) 075021. 